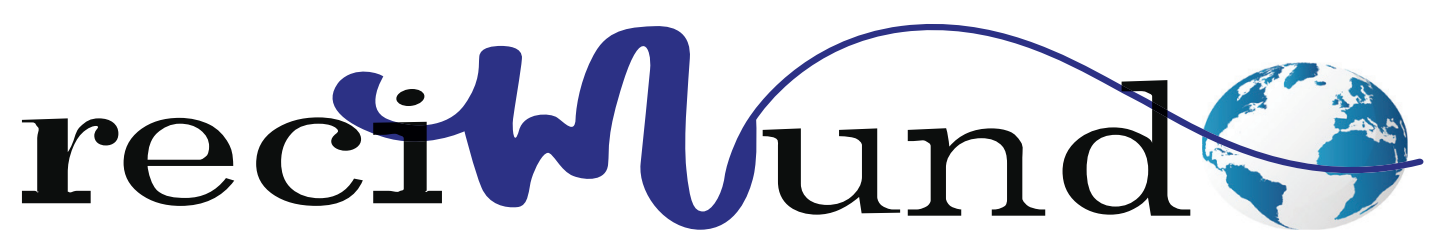

Revista Científica Mundo de la Investigación y el Conocimiento

DOI: 10.26820/recimundo/4.(1).enero.2020.306-314

URL: http://recimundo.com/index.php/es/article/view/727

EDITORIAL: Saberes del Conocimiento

REVISTA: RECIMUNDO

ISSN: 2588-073X

TIPO DE INVESTIGACIÓN: Artículo de Revisión

CÓDIGO UNESCO: 3202 Epidemiología; 3201.10 Pediatría

PAGINAS: $306-314$

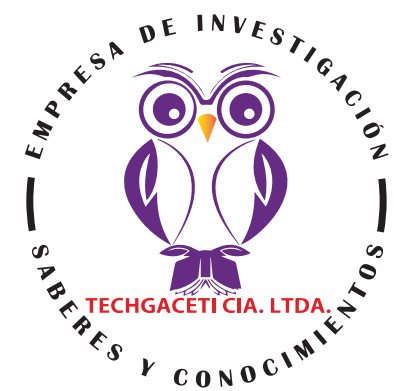

\title{
Principales factores de riesgos de morbilidad y mortalidad en neonatos pre términos extremos. Hospital IESS Babahoyo durante el periodo 2019
}

Main morbidity and mortality risk factors in preterm infants preterm terms. IESS Babahoyo Hospital during the 2019 period

Principales factores de riesgos de morbilidad y mortalidad en neonatos pre términos extremos. Hospital IESS Babahoyo durante el periodo 2019

Gianella Fabiola Encalada Marín'; Karem Lisseth Aguirre Realpe'; Aliz Janeth Calero Correa ${ }^{3}$

RECIBIDO: 18/09/2019 ACEPTADO: 29/10/2019 PUBLICADO: 31/01/2020

1. Médico; Investigador Independiente; Guayaquil, Ecuador; gianellafabiolaencaladamarin@gmail.com; (D) https://orcid.org/0000-0002-9235-0141

2. Médico; Investigador Independiente; Guayaquil; kaguirrerczs5@gmail.com; (D) https://orcid.org/0000-00029129-2322

3. Médico; Investigador Independiente; Guayaquil, Ecuador, alizcalero@hotmail.com; (D) https://orcid.org/00000001-7317-0500

\section{CORRESPONDENCIA}

Gianella Fabiola Encalada Marín

gianellafabiolaencaladamarin@gmail.com

Guayaquil, Ecuador

๑) RECIMUNDO; Editorial Saberes del Conocimiento, 2020 


\section{RESUMEN}

La Organización Mundial de la Salud define el parto pre término como aquel que acontece antes de las 37 semanas completas o 259 días desde la fecha de última regla. En la actualidad, este constituye la principal causa de morbilidad y mortalidad perinatales. La prematuridad continúa siendo la causa más frecuente de muerte neonatal y representa aproximadamente $3 / 4$ de la mortalidad en esta etapa, así como cerca de la mitad de la morbilidad neurológica a largo plazo. La sepsis inicialmente se diagnostica por la presencia de signos y síntomas, a lo que se considera como sepsis probable. Para que deje de serlo y se convierta en confirmada, se usa como determinante: la realización y análisis de resultado a un cultivo. Se clasifica a la sepsis en 2 grupos: sepsis temprana (primeros 7 días de vida) y sepsis tardía (posterior a los 7 días de vida). El síndrome de dificultad respiratorio es un cuadro respiratorio agudo que afecta casi exclusivamente a los recién nacidos pre término, la inmadurez del pulmón del pre término no es solamente bioquímica, déficit de surfactante pulmonar, sino también morfológica y funcional, debido a que el desarrollo pulmonar aún no se ha completado en estos niños inmaduros. El diagnóstico se basa en los antecedentes, datos clínicos y en el aspecto radiográfico de los pulmones, si bien la radiografía puede no reflejar la intensidad de la afectación pulmonar, sobre todo cuando el neonato recibe asistencia respiratoria. Los resultados obtenidos del estudio demuestran una baja mortalidad. Se usó una metodología descriptiva, con un enfoque documental, es decir, revisar fuentes disponibles en la red, con contenido oportuno y relevante para dar respuesta a lo tratado en el presente artículo. Se realizó un estudio descriptivo observacional de corte transversal, en el que se incluyeron todos los recién nacidos pre términos que acudieron al centro hospitalario durante el periodo de estudio.

Palabras clave: Sepsis, Neonatal Pretérmino, Síndrome de Dificultad Respiratoria, Mortalidad, UCIN.

\section{ABSTRACT}

The World Health Organization defines preterm birth as one that occurs before the full 37 weeks or 259 days from the date of last rule. At present, this constitutes the main cause of perinatal morbidity and mortality. Prematurity remains the most frequent cause of neonatal death and represents approximately aproximadamente of mortality at this stage, as well as about half of the long-term neurological morbidity. Sepsis is initially diagnosed by the presence of signs and symptoms, which is considered as probable sepsis. To stop being so and become confirmed, it is used as a determinant: the performance and analysis of results to a crop. Sepsis is classified into 2 groups: early sepsis (first 7 days of life) and late sepsis (after 7 days of life). The respiratory distress syndrome is an acute respiratory condition that affects almost exclusively preterm infants, the immaturity of the preterm lung is not only biochemical, pulmonary surfactant deficit, but also morphological and functional, because lung development It has not yet been completed in these immature children. The diagnosis is based on the history, clinical data and radiographic appearance of the lungs, although the x-ray may not reflect the intensity of the lung involvement, especially when the newborn receives respiratory assistance. The results obtained from the study demonstrate low mortality. A descriptive methodology was used, with a documentary approach, that is, to review sources available on the network, with timely and relevant content to respond to what is discussed in this article. An observational cross-sectional descriptive study was carried out, which included all pre-term newborns who attended the hospital during the study period.

Keywords: Sepsis, Preterm Neonatal, Respiratory Difficulty Syndrome, Mortality, NICU.

\section{RESUMO}

A Organização Mundial da Saúde define nascimento prematuro como aquele que ocorre antes das 37 semanas ou 259 dias a partir da data da última regra. Atualmente, isso constitui a principal causa de morbimortalidade perinatal. A prematuridade continua sendo a causa mais frequente de morte neonatal e representa aproximadamente aproximadamente a mortalidade nesta fase, bem como cerca de metade da morbidade neurológica a longo prazo. A sepse é inicialmente diagnosticada pela presença de sinais e sintomas, que é considerada provável sepse. Para deixar de ser assim e se confirmar, é utilizado como determinante: o desempenho e a análise dos resultados de uma cultura. A sepse é classificada em 2 grupos: sepse precoce (primeiros 7 dias de vida) e sepse tardia (após 7 dias de vida). A síndrome do desconforto respiratório é uma condição respiratória aguda que afeta quase exclusivamente prematuros, a imaturidade do pulmão prematuro não é apenas bioquímica, déficit de surfactante pulmonar, mas também morfológica e funcional, porque o desenvolvimento pulmonar ainda não foi concluído nessas crianças imaturas. O diagnóstico é baseado na história, nos dados clínicos e na aparência radiográfica dos pulmões, embora a radiografia possa não refletir a intensidade do envolvimento pulmonar, principalmente quando o recém-nascido recebe assistência respiratória. Os resultados obtidos no estudo demonstram baixa mortalidade. Utilizou-se uma metodologia descritiva, com abordagem documental, ou seja, para revisar as fontes disponíveis na rede, com conteúdo oportuno e relevante para responder ao que é discutido neste artigo. Foi realizado um estudo descritivo transversal observacional, que incluiu todos os recém-nascidos pré-termo que compareceram ao hospital durante o período do estudo.

Palavras-chave: Sepse Neonatal Pré-termo, Síndrome de Dificuldade Respiratória, Mortalidade, UTIN. 


\section{Introducción}

La mortalidad neonatal es uno de los indicadores de salud más importantes de un país, mide el grado de desarrollo de una nación y el estado de salud de la población; por lo tanto, medirla colabora con la planeación de políticas y estrategias específicas de salud. La Organización Mundial de la Salud señala que en el mundo fallecen cuatro millones de recién nacidos por año. De esta cifra, $75 \%$ durante la primera semana de vida, y 25 a 45\% en el primer día de vida. La mortalidad neonatal es causada principalmente por procesos infecciosos en un 36\% y a la prematuridad en un 28\% (Cárdenas-Méndez, Marzo 2012).

La incidencia de sepsis neonatal en el mundo desarrollado se encuentra entre 0,6\% y el $1,2 \%$ de todos los nacidos vivos, pero en el mundo en desarrollo puede alcanzar entre el 20 y el $40 \%$ de todos los nacidos vivos (González-Caraballo, Agosto 2006). Esta patología representa un reto para los servicios de neonatología del mundo; es imprescindible un diagnostico precoz, específico, y un tratamiento antimicrobiano adecuado que nos permita reducir los índices de morbimortalidad, especialmente en los sectores más vulnerables de la población.

El síndrome de dificultad respiratoria presenta un cuadro clínico que afecta casi exclusivamente a pre términos y a hijos de madre diabética. La sintomatología se inicia inmediatamente posterior al nacimiento y va aumentando de intensidad entre las 48-72 horas para luego regresar en el curso de una semana o diez días. Los síntomas más comunes son: quejido ex piratorio, retracción esternal y subcostal, polipnea y tianosis. La auscultación pulmonar revela menor entrada de aire a los pulmones, disminución del murmullo vesicular y a veces crépitos.

\section{Metodología}

Esta investigación está dirigida al estudio del tema "Principales factores de riesgos de morbilidad y mortalidad en neonatos pre términos extremos. Hospital IESS Babahoyo durante el periodo 2019". Para realizarlo se usó una metodología descriptiva, con un enfoque documental, es decir, revisar fuentes disponibles en la red, como google académico, con contenido oportuno y relevante desde el punto de vista científico para dar respuesta a lo tratado en el presente artículo y que sirvan de inspiración para realizar otros proyectos. Las mismas pueden ser estudiadas al final, en la bibliografía. Se realizó un estudio descriptivo observacional de corte transversal, en el que se incluyeron todos los recién nacidos pre términos que acudieron al centro hospitalario durante el periodo de estudio.

\section{Resultados}

La Organización Mundial de la Salud define el parto pre término como aquel que acontece antes de las 37 semanas completas o 259 días desde la fecha de última regla. En la actualidad, este constituye la principal causa de morbilidad y mortalidad perinatales. La prematuridad continúa siendo la causa más frecuente de muerte neonatal y representa aproximadamente $75 \%$ de la mortalidad en esta etapa, así como cerca de la mitad de la morbilidad neurológica a largo plazo (Cabanillas-López, 2014).

En América Latina nacen en promedio unos 12 millones de niños cada año: 400000 mueren antes de cumplir 5 años; 270000 en el primer año de vida; 180000 durante el primer mes y 135000 por parto prematuro. La situación es aún más grave en los prematuros extremos (menos de 32 semanas de embarazo), entre quienes un $20 \%$ no sobrevive el primer año, $60 \%$ de los supervivientes tiene discapacidades neurológicas (problemas de lenguaje y aprendizaje, trastorno por déficit de atención, dificultades socioemocionales, deterioro sensorial, visual y auditivo, retraso mental y parálisis cerebral) (Cabanillas-López, 2014).

Estudios han determinado que el parto pre término tiene un carácter multifactorial: intervienen enfermedades maternas, emba- 
razos múltiples originados por técnicas de reproducción asistida e inductores de la ovulación, infecciones, estilos de vida y diferentes situaciones sociales, edad de la madre, la colonización del tracto genital femenino, la respuesta inflamatoria, la rotura prematura de membranas. El inicio del parto se origina por la liberación de prostaglandinas localmente en la inter fase corion-decidual que actúan sobre el miometrio y provoca las contracciones uterinas (Couto-Nápoles, 2014).

La Organización Mundial de la Salud señala que en el mundo fallecen cuatro millones de recién nacidos anualmente. De esta cifra, $75 \%$ durante la primera semana de vida, y 25 a $45 \%$ en el primer día de vida. La mortalidad neonatal es causada principalmente por procesos infecciosos en un $36 \%$ y a la prematuridad en un $28 \%$. En Sudamérica, se han cuantificado factores de riesgo para la sepsis neonatal: bajo peso de nacimiento con un $10 \%$, el género masculino tiene una incidencia de $63 \%$ de los casos de sepsis, la ruptura prolongada de membrana en un $20 \%$ y post término 34\% (Couto-Nápoles, 2014).

La sepsis inicialmente se diagnostica por la presencia de signos y síntomas, a lo que se considera como sepsis probable. Para que deje de serlo y se convierta en confirmada, se usa como determinante: la realización y análisis de resultado a un cultivo. Se clasifica a la sepsis en 2 grupos (González-Caraballo, Agosto 2006):

- Sepsis temprana (primeros 7 días de vida): es probable que se encuentren antecedentes de problemas perinatales, rotura prematura de membrana, parto prolongado, amnionitis, fiebre materna, hipertensión/ pre eclampsia, prematurez y asfixia intrauterina. Sin embargo en ocasiones como puede ocurrir en la sepsis por EGB, no hay ningún antecedente patológico que pueda alertar al médico y se presentan signos clínicos graves en un niño que

\section{nació de parto normal}

- Sepsis tardía (luego de los primeros 7 días), algunos signos y cuadro clínico: disminución de la vitalidad, menor aumento de peso, o inestabilidad térmica, rechazo del alimento, letargia o irritabilidad, ictericia con predominio directo, taquipnea, apnea (en especial en prematuros), vómitos, distensión abdominal y diarrea. Las convulsiones, el escleredema y el shock son sumamente tardíos.

La producción de la infección neonatal, se asocia a considerar 3 procesos principales (Moraes-Pimienta, Septiembre 2009):

a) Infección intrauterina: implica una infección materna generalmente solapada o con signos clínicos inespecíficos. La vía de infección fetal más frecuente es la hematógena, a través de la placenta.

b) Infección adquirida durante el parto: se relaciona con corioamnionitis, infección urinaria materna y en especial con la rotura prematura o prolongada de las membranas ovulares.

c) Infección postnatal: está condicionada por múltiples factores, siendo el fundamental la prematurez y bajo peso al nacer. Otros aspectos a tener en cuenta: características inmunológicas, asfixias, patologías subyacentes no infecciosas, permanencia prolongada en el hospital, procedimientos especiales, intervenciones quirúrgicas, alimentación con fórmulas lácteas y deficientes medidas de profilaxis y control en los servicios hospitalarios.

\section{Diagnóstico}

Uno de los aspectos importantes para el manejo de la sepsis es la identificación bacteriológico del gérmen en sangre o LCR, pero habitualmente el médico no tiene sus resultados hasta pasadas las 24 o 48 horas, lo cual significa una demora que no es permisible para el comienzo del tratamiento (Fernández-Guevara, 2011):

1. Técnicas microbiológicas: el aislamien-

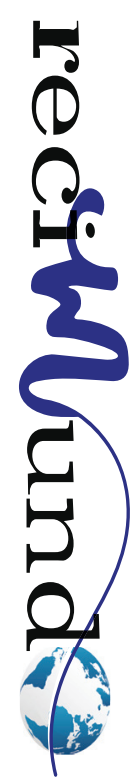


to de un germen en muestras tomadas de los diferentes fluidos corporales, constituye el método de diagnóstico más exacto y confirmatorio.

- Hemocultivo: suele bastar 0,5 ml de sangre venosa.

- Punción lumbar: es un excelente y rápido método que nos permite a través del análisis del LCR, determinar si existe meningitis.

- Uro cultivo: se debe hacer por punción vesical supra púbica. La observación de gérmenes en el examen directo o el desarrollo de cualquier número de colonias es confirmatorio de infección.

- Otros cultivos: punción intra articular, punción de pústulas, celulitis, drenaje de absceso.

- Detección de microorganismos por técnicas microbiológicas: la contra inmuno electroforesis es un método que identifica antígenos bacterianos.

- Recuento de leucocitos y neutrófilos: el índice que resulta del cociente entre el número de neutrófilos inmaduros y el número total de neutrófilos es un elemento utilizado asiduamente para el diagnóstico de sepsis. Se considera normal un valor mayor a 0,2 (o mayor a 0,3 en las primeras 24 horas).

- Microeritrosedimentación: la eritrosedimentación se puede realizar mediante la recolección de una muestra sanguínea en capilares y la sedimentación de los eritrocitos durante una hora.

- Proteína C reactiva (PCRe) e interleuquina 8: la PCRe es una excelente prueba aunque no útil en el diagnóstico temprano mientras, que la interleuquina 8 producida por los monocitos-macrófagos, aumentan pronto en el curso de las infecciones bacterianas neonatales. Estudios recientes han demostrado que la combinación de ambos métodos diag- nósticos han reducido el uso innecesario de antibióticos en los recién nacidos.

- Alteraciones citoplasmáticas de los neutrófilos: las más frecuentes son granulaciones tóxicas, vacuolización y cuerpos de Döhle. Las granulaciones tóxicas son las de mayor valor, si son numerosas indica en general infección bacteriana.

- Plaquetopenia: la disminución del número de plaquetas por debajo de 150.000/ $\mathrm{mm}^{3}$ es un signo en sepsis bacterianas como en infecciones virales.

- Examen del líquido de aspirado gástrico: descarta que no se encuentren gérmenes ni polimorfonucleares en el jugo gástrico, en cuyo caso el diagnostico de sepsis es poco probable.

2. Exámenes de diagnóstico por imágenes (Demestre-Raspall, Octubre 2009):

- Radiografía de tórax: su utilización es de rigor para el diagnóstico de neumonía, en el recién nacido, la neumonía suele ser multifocal, por lo que las imágenes muestran opacidades e infiltrados bilaterales con predominio a veces en algún sector.

- Radiografía de abdomen: debe ser utilizado siempre que se tenga evidencias clínicas o sospecha de infección intestinal o de enterocolitis necrotizante.

- Radiografía de huesos: útil para el seguimiento de las lesiones en infecciones osteoarticulares.

- Ecografía: es útil cuando se sospecha patología abdominal, principalmente absceso intra hepáticos o renales. La ecoencefalografía es útil para el diagnóstico y seguimiento de recién nacidos con meningitis.

- Tomografía computarizada: su mayor uso es el diagnostico de absceso cerebral y en patología pulmonar.

- Resonancia magnética nuclear: es útil 
en infecciones del sistema nervioso central y de la columna vertebral.

\section{Síndrome de Dificultad Respiratoria}

Es un cuadro respiratorio agudo que afecta casi exclusivamente a los recién nacidos pre término. La inmadurez del pulmón del pre término no es solamente bioquímica, déficit de surfactante pulmonar, sino también morfológica y funcional, debido a que el desarrollo pulmonar aún no se ha completado en estos niños inmaduros. El pulmón con déficit de surfactante es incapaz de mantener una aireación y un intercambio gaseoso adecuados (López-Valls, 2008).

\section{Diagnóstico}

El diagnóstico se basa en los antecedentes, datos clínicos y en el aspecto radiográfico de los pulmones, si bien la radiografía puede no reflejar la intensidad de la afectación pulmonar, sobre todo cuando el neonato recibe asistencia respiratoria. En el fluir de la enfermedad aparecen los cambios típicos: disminución del volumen pulmonar, opacificación difusa de los campos pulmonares con un moteado fino de aspecto de vidrio esmerilado y presencia de bronco grama aéreo (Rizzardini, 1993). Hay que evaluar la presencia de complicaciones como enfisema intersticial, neumotórax o, con el tiempo, evolución a una enfermedad pulmonar crónica. El cuidado de estos pacientes debe efectuarse en unidades de cuidados intensivos neonatales.

Cifras de manifestaciones clínicas de pre término en el Hospital IESS Babahoyo durante el periodo 2019

Se realizó un estudio descriptivo observacional de corte transversal, en el que se incluyeron todos los recién nacidos pre término peso al nacer desde 1.500 grs a 3.000 grs y menos de 37 semanas de estado de gestación, en el período comprendido entre enero hasta diciembre de 2019, en el Hospital IESS Babahoyo. Se excluyeron aquellos que no cumplieron con lo anterior, y en los que se encontraron limitantes para la investigación (pacientes cuyas historias clínicas no contenían datos, tanto maternos como perinatales, de interés para esta investigación). Con estos criterios quedó establecida la población de estudio con 415 neonatos pre término.

Se utilizaron, como fuente primaria de información, las historias clínicas de las madres y los recién nacidos pre término que acudieron a consulta, la recolección de datos se realizó a través de una encuesta no estructurada elaborada para este fin. Las variables de estudio fueron: afecciones maternas, edad gestacional, peso al nacer del recién nacido, sexo, modo de nacimiento, ingreso, egreso y diagnóstico clínico.

De forma general se aplicaron medidas de resumen para datos cualitativos en variables de este orden, especialmente a las referidas al diagnóstico clínico: sepsis, síndrome de dificultad respiratoria y mortalidad. Los resultados obtenidos se resumen en la siguiente Tabla $N^{\circ} 1$. Los resultados del estudio solo se publicarán con fines científicos y se mantendrán los datos personales en estricta confidencialidad. 


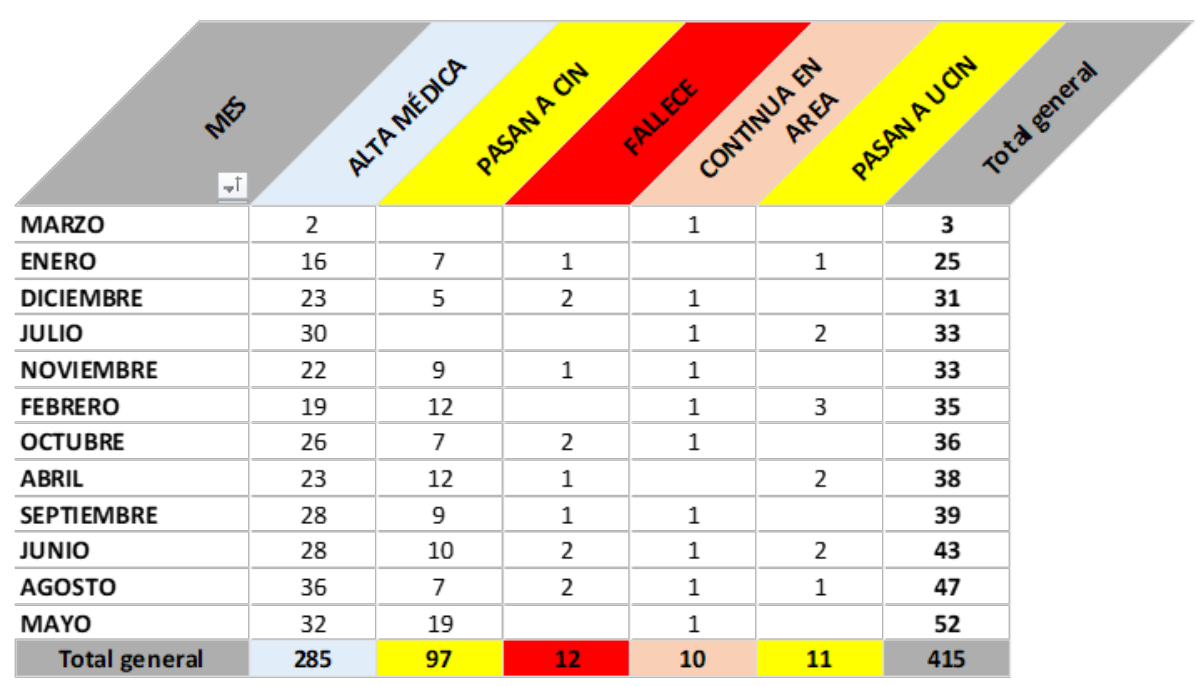

Figura 1. Resultados de la Atención a Recién Nacidos Pre términos en el Hospital IESS Babahoyo durante el periodo 2019

Fuente: Los autores 2020

Se observa que el motivo de consulta fueron las infecciones con mayor prevalencia, diagnosticadas en la mayoría de datos obtenidos, (80,3 \%). En estos, el comportamiento específico de la sepsis de comienzo precoz (antes de las 72 horas) fue de 41,3 $\%$, mientras que el 58,73 \% presentó la de comienzo tardío. En relación con el universo, $38 \%$ pacientes fueron diagnosticados con sepsis de comienzo tardío, otra enfermedad muy importante por su repercusión en el neonato, y que afectó al 31,7 \% de los pacientes, fue la enfermedad de membrana hialina o síndrome de dificultad al respirar.

Cabe destacar que la buena práctica médica contribuyo a la disminución de la mortalidad de los recién nacidos pre término, situándose en $2.66 \%$ mientras que los pacientes evaluados del estudio dirigidos a la UCIN represento un $2.5 \%$.

También se puede apreciar en la figura 1 la incidencia de los resultados del estudio, fallecieron 12 pacientes. La sepsis fue la responsable del 34,2 \% de las defunciones; dentro de esta, la de comienzo tardío fue la más frecuentemente encontrada como causa directa de muerte (69,2\%), lo cual guarda total correspondencia con la morbilidad descrita. La letalidad para la sepsis global fue de $17 \%$. La tasa de sepsis, según el universo estudiado, fue de $12,35 \%$. Le siguió en orden de frecuencia el síndrome de dificultad respiratoria 21,4\%. Al analizar la tasa de fallecidos por esta afección, según el total de nacidos vivos, se encontró que esta fue de $5,0 \%$ y la letalidad de $14,5 \%$. 
Distribución de pacientes pretérmino

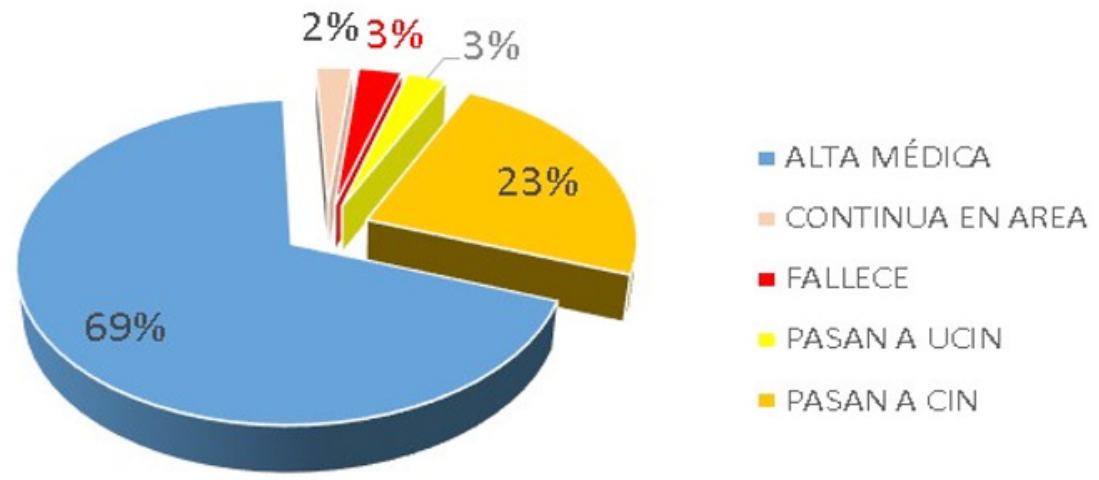

Figura 2. Distribución de 415 recién nacidos pre término en el Hospital IESS Babahoyo durante el periodo 2019

Fuente: Los autores 2020

\section{Conclusión}

Los niños pre términos, son más perceptibles a mostrar traumatismos durante el parto que los fetos a término; tienen más probabilidad de sufrir daño en los tejidos blandos, daño neurológico y hemorragia intracraneal traumática que los niños a término. A medida que se incrementó la edad gestacional y el aumento de peso, lo que se explica porque desde finales del segundo trimestre de gestación, comienza una ganancia gradual del peso fetal a expensas de la acumulación de grasas y glucógeno.

En fuentes consultadas, la incidencia de neonatos con síndrome de dificultad respiratoria muestra igual comportamiento, pues se considera que luego de las 34 semanas de edad de gestación la morbilidad y mortalidad relacionadas con la madurez se minimiza, razón por la cual, a pesar de los beneficios que engloba la prolongación de la gestación, se valora la interrupción una vez que la embarazada alcanza ese momento. Incluso ha sido demostrado que la administración de corticoides a la madre disminuye la incidencia de esta patología, la mortalidad y la tasa de hemorragia intra ventricular. Este tratamiento produce no sólo un aumento sino también la remodelación y maduración de la estructura elástica pulmonar.
En relación con la elevada frecuencia de la sepsis de comienzo tardío, esto está en correspondencia con la literatura, que reporta mayores índices de sepsis luego del tercer día de vida. En la distribución por grupos de edades esta entidad predominó entre las 30-31,6 semanas, período en el cual ocurrió un mayor número de nacimientos de pacientes con pesos entre 1.900 grs a 2.400; sin embargo, luego de las 34 semanas, la incidencia de esta afección decreció. Esto obedece, además de a la relación directa entre edad gestacional y madurez, la presencia de la hemorragia intra ventricular se relaciona además con el no uso de esteroides (pues ellos también inducen la maduración del sistema nervioso central) y con el tratamiento a este tipo especial de pacientes. Destaca también una disminución significativa de la mortalidad de la población estudiada, menor a lo reflejado por entes para países subdesarrollados.

\section{Bibliografía}

Cabanillas-López. (2014). Características de la sepsis neonatal en el Hospital Almanzor Aguinaga Asenjo durante los años 2011-2012. Revista Cuerpo Médico HNAAA , 7(4), 6 - 10. Obtenido de http://www.cmhnaaa.org.pe

Cárdenas-Méndez. (Marzo 2012). Morbilidad y mortalidad del recién nacido pretérmino menor de 1 500 gramos. Revista Cubana de Pediatria, 48(1).

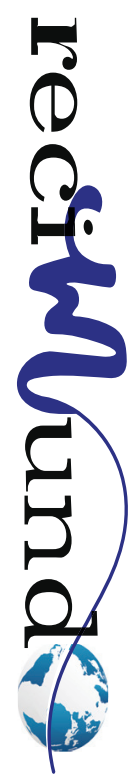




\section{Obtenido de http://scielo.sld.cu}

Couto-Nápoles. (2014). Repercusión del parto pretérmino en la morbilidad y mortalidad perinatales. MEDISAN, vol. 18, núm. 6, 2014,, 18(6), 852 - 858. Obtenido de https://www.redalyc.org

Demestre-Raspall. (Octubre 2009). Prematuros tardíos: una población de riesgo infravalorada. Anales de Pediatría Volume 71, Issue 4, October 2009, Pages 291-298, 71(4), 291 - 298. Obtenido de https://www.sciencedirect.com

Fernández-Guevara. (2011). Factores de riesgo asociados a mortalidad en neonatos menores de 1500 g utilizando la escala CRIB II. Boletín Medico Hospital Infantil, 68(5), 356 - 362. Obtenido de http:// www.scielo.org.mx
González-Caraballo. (Agosto 2006). SEPSIS NEONATAL Y PREMATUREZ. Revista de Posgrado de la Vla Cátedra de Medicina - No 160 - Agosto 2006, 160(1), 22 - 28. Obtenido de https://med. unne.edu.ar

López-Valls. (2008). Síndrome de dificultad respiratoria. Protocolos actualizados al año 2008., 305 309. Obtenido de https://www.aeped.es

Moraes-Pimienta. (Septiembre 2009). Morbilidad en pretérminos tardíos: estudio prospectivo caso control multicéntrico. Archivo de Pediatrría Uruguay vol.80 no.3 Montevideo set. 2009, 80(3). Obtenido de http://www.scielo.edu.uy

Rizzardini, M. (1993). Sindrome de dificultad respiratoria del recien nacido. Revista Chilena Pediatria, 44(6), 531 - 539. Obtenido de https://scielo. conicyt.cl

\section{CITAR ESTE ARTICULO:}

Encalada Marín, G., Aguirre Realpe, K., \& Calero Correa, A. (2020). Principales factores de riesgos de morbilidad y mortalidad en neonatos pre términos extremos. Hospital IESS Babahoyo durante el periodo 2019. RECIMUNDO, 4(1), 306-314. doi:10.26820/recimundo/4.(1).enero.2020.306-314 